REVUE DE L'INSTITUT

FRANC AIS D'HISTOIRE

EN ALLEMAGNE

\section{Revue de l'IFHA}

Revue de l'Institut français d'histoire en Allemagne

$6 \mid 2014$

IFHA 6

\title{
Colloque « Une histoire croisée de l'ethnologie : approche nationale et transnationale de l'histoire de l'ethnologie »
}

Paris, EHESS, 16 et 17 juin 2014

Jean-Louis Georget

\section{(2) OpenEdition}

\section{Journals}

Édition électronique

URL : http://journals.openedition.org/ifha/7993

DOI : 10.4000/ifha. 7993

ISSN : 2198-8943

Éditeur

IFRA - Institut franco-allemand (sciences historiques et sociales)

Édition imprimée

Date de publication : 31 décembre 2014

ISSN : 2190-0078

\section{Référence électronique}

Jean-Louis Georget, «Colloque « Une histoire croisée de l'ethnologie : approche nationale et

transnationale de l'histoire de l'ethnologie » », Revue de l'IFHA [En ligne], 6 | 2014, mis en ligne le 31

décembre 2014, consulté le 03 mai 2019. URL : http://journals.openedition.org/ifha/7993 ; DOI :

$10.4000 /$ ifha. 7993

Ce document a été généré automatiquement le 3 mai 2019.

(CIFHA 


\section{Colloque « Une histoire croisée de l'ethnologie: approche nationale et transnationale de l'histoire de l'ethnologie»"}

Paris, EHESS, 16 et 17 juin 2014

Jean-Louis Georget

1 Après une courte présentation du colloque par Hélène Ivanoff et Martin Mourre, Clément Zobel (Paris 8) a présenté, dans le cadre du premier atelier intitulé « Approche comparée de la construction d'un savoir africaniste en Europe/Die Herausbildung von Wissensbeständen über Afrika in Europa » un exposé intitulé «Culture et civilisation chez Leo Frobenius et Maurice Delafosse ». Sa démarche consistait à mettre en relation les œuvres de Leo Frobenius, pionnier de la recherche africaniste allemande, et celles de son homologue français Maurice Delafosse. Malgré leurs formations différentes, ces deux auteurs menaient des projets de comparaison interculturelle similaires. Leurs travaux, ayant pour but une valorisation humaniste des civilisations africaines, s'inscrivaient dans un paradigme commun: l'essentialisme culturaliste, dont nous pouvons faire remonter les origines à la critique allemande de la philosophie universaliste des Lumières. Dans leur souci de délimiter des cultures et civilisations, Frobenius et Delafosse faisaient référence à la préhistoire, au concept de mentalités collectives, et, même s'ils s'en défendaient, aux catégories raciales. La différence entre observateur et observé qui était ainsi constituée posait la problématique d'une construction apolitique du sujet ethnologique.

2 Puis Isabella Atger (Université Paris 3 Sorbonne Nouvelle) a exploré l'œuvre des deux africanistes Leo Frobenius et Marcel Griaule dans une discipline en construction. À travers l'exemple de ces deux africanistes, respectivement les premiers dans leur pays à partir sur le terrain, a été observée l'évolution de l'ethnologie du début du XX ${ }^{e}$ siècle. Elle était marquée par son désir de s'affirmer comme science et de ce fait, par sa recherche d'objectivité dans les méthodes utilisées sur le terrain et dans la retranscription écrites 
des observations. L'exposé s'est arrêté sur les conséquences induites par l'utilisation des fiches documentaires sur le terrain et le rôle du récit, son acception double de métacode textuel et de narration, sur le travail des deux hommes qui, vers la fin de leur vie, attestaient la valeur des cultures qu'ils avaient découvertes et contestaient le stigmate de primitivité dont étaient affublés les peuples africains.

Verena Baldwin (Österreichen Akademie der Wissenschaften) et Anita Dick (université de Vienne) ont ensuite évoqué "Walter Hirschberg et la (re-)construction d'un savoir africain à Vienne (1928-1945)». Walter Hirschberg (1906-1994), professeur à l'université de Vienne, fut titulaire de la deuxième chaire d'ethnologie à l'Institut für Völkerkunde de 1962 à 1975. Dans son travail scientifique des années 1928 à 1945, Hirschberg se voua essentiellement à l'ethnographie de l'Afrique et à des thèmes d'ethnologie européenne. En partant de ses travaux, il est possible de montrer comment un savoir sur l'Afrique s'est construit à Vienne, après la fondation de l'Institut d'ethnologie et à l'époque du nazisme. En Autriche, l'accession au pouvoir du parti national socialiste en 1938 a été positive pour la carrière de Walter Hirschberg, qui - membre de la NSDAP encore illégale - a été engagé au Museum für Völkerkunde à Vienne et a ainsi pu passer son habilitation en 1939. L'Anschluß de l'Autriche à l'Allemagne nazie lui avait donc aussi profité, et le renvoi du Père Wilhelm Koppers à cause de cette évolution politique est également un fait qui lui a été favorable. Lorsque l'ethnologue de musée berlinois, Hermann Baumann, se vit attribuer la chaire laissée vacante par le départ de Wilhelm Koppers, une nouvelle ère débuta, celle d'un diffusionnisme laïque, qui permettait en autres choses de justifier la nécessité - comme objectif politique - de la création d'« un empire colonial d'Afrique centrale » sous la direction de l'Allemagne.

4 L'après-midi s'est achevée par des lectures croisées d'Emmanuelle Sibeud (Paris 8) et Hans Peter Hahn (université de Francfort) sur le thème "Africanisme versus panafricanisme autour de $1900 »$. Les deux chercheurs ont débattu sur les parcours de Bénito Sylvain et W. E. Dubois et leur rôle dans l'émergence du panafricanisme au début $\mathrm{du}$ XXe siècle.

5 L'ensemble des participants se sont ensuite rendus à la présentation de l'exposition « Leo Frobenius (1873-1938) et l'art rupestre africain ", introduite par Joachim Umlauf (Institut Goethe, Paris) et Karl-Heinz Kohl (Institut Frobenius, Francfort). János Riesz (université de Bayreuth) a ensuite fait une conférence sur le thème du «mythe de l'Atlantide au cœur de la vision historique de Leo Frobenius "

6 La suite du colloque avait lieu le mardi 17 juin.

7 Dans le premier atelier intitulé « Histoire croisée de l'anthropologie en Europe et aux Etats-Unis », Jean-Louis Georget (Institut français d'histoire en Allemagne) et Richard Kuba (Institut Frobenius) ont présenté un regard croisé sur l'ethnologie en France et en Allemagne. Des travaux sur l'histoire de l'ethnologie existent côté allemand et français, mais une histoire croisée de l'ethnologie en France et en Allemagne reste à écrire. Les chercheurs se sont peu rencontrés. Ceci tient au fait d'une part que la plupart des travaux sont inaccessibles d'un point de vue linguistique à l'autre partie scientifique et que les chercheurs français n'ayant pas accès à la langue allemande travaillent donc sur un corpus limité. Les travaux français, y compris sur Frobenius, sont parfois inconnus en Allemagne, alors que les travaux des grands anthropologues français ont été abondamment traduits. D'autre part, le statut de l'ethnologie n'est pas le même en France et en Allemagne sur plusieurs plans : elle est moins valorisée en Allemagne, ayant une moindre notoriété, elle est coupée en deux (ethnologie européenne, ethnologie allogène) 
et elle n'a pas la même matrice disciplinaire. À l'instar d'autres disciplines, l'ethnologie française avait cherché à la fin des années 1970 à se rapprocher de son homologue allemande pour des raisons multiples, scientifiques en premier lieu, puisque l'étrangeté que constituait la double onomastique allemande, qui distinguait l'ethnologie appliquée à l'Allemagne sous le terme de Volkskunde et l'ethnologie concernant les peuples colonisés sous le vocable de Völkerkunde, n'était que peu connue en France, y compris des cercles de spécialistes. La filiation duale, celle du creuset des ethnologies française et anglaise d'une part, plus orienté vers le modèle de la Völkerkunde, et du modèle allemand et américain d'autre part, plus orienté vers la Volkskunde du fait des particularités territoriales, qui passait notamment par les figures de Wilhelm Heinrich Riehl et de Franz Boas, abondamment accessibles l'un et l'autre outre-Atlantique, laissait la porte ouverte à des considérations prometteuses. La première phase de rapprochement disciplinaire passa par la médiation des acteurs de l'École des Hautes Etudes en Sciences Sociales d'une part et de l'institut Ludwig-Uhland de Tübingen d'autre part, qui initièrent une nouvelle phase des rapports franco-allemands dans le domaine et qui permirent un rapprochement notable des idées et des concepts, prolongé aujourd'hui par de nouvelles initiatives.

Christine Laurière (EHESS) a ensuite traité de la question du musée ethnographique au cœur de l'institutionnalisation de l'ethnologie en France. L'ethnologie française dans les années 1920-1940, contrairement à ce qui se passe aux États-Unis et en Angleterre, reste intimement liée au musée d'ethnographie au moins jusque dans les années 1950. Jeune discipline universitaire (l'Institut d'ethnologie est fondé en 1925), l'ethnologie s'impose avec force sur la scène intellectuelle et culturelle grâce à l'entreprise de rénovation du musée d'ethnographie du Trocadéro que mènent Paul Rivet et Georges Henri Rivière à partir de 1928. Entre 1925 et 1940, une centaine de missions ethnographiques est organisée, principalement dans les territoires sous domination impériale. La relation entre les jeunes ethnologues de l'Institut d'ethnologie et le Trocadéro est consubstantielle: à tous s'imposent l'impératif absolu de la collecte d'objets ethnographique, qui va de pair avec le paradigme de l'ethnographie de sauvetage, dans la mesure où tous sont convaincus d'assister aux derniers feux des sociétés traditionnelles. Sans n'avoir jamais été une science coloniale, l'ethnologie profite objectivement, pragmatiquement, des conditions offertes par l'Empire pour aider la professionnalisation des jeunes élèves.

Après une discussion, Marie France Chevron (université de Vienne) a exposé les débuts de l'ethnologie en pays de langue allemande à partir de l'exemple de Vienne. La présentation était basée sur les résultats d'une recherche menée en 2006 et 2007 dans les archives de la Société d'Anthropologie à Vienne. À une première phase formative de l'ethnologie suit une phase de consolidation progressive caractérisée par les efforts des chercheurs pour obtenir l'établissement de l'ethnographie à l'Université. Cette période euphorique fut marquée par des échanges intenses au sein de la Société. Le champ anthropologique différencié certes mais aussi encore très diffus permet ou parfois entrave la formation des différentes disciplines: A côté des sociétés et musées (Verein/ Museum für Volkskunde, 1894/95, et Museum für Völkerkunde, 1928) mais aussi de l'université (1913 et 1929), l'Académie des Sciences est un des espaces où l'ethnologie s'établit à Vienne, toujours avec un regard tourné en direction de l'Allemagne.

10 Glenn Penny (University of Iowa) a ensuite disserté sur la vision d'Adolf Bastian, Franz Boas et la politique muséographique allemande. Cette contribution a défendu la thèse que l'anthropologie de Franz Boas s'inscrit dans la continuité du projet qui a guidé les musées 
ethnographiques allemands au XIXe siècle. Ce projet était basé dans une large mesure sur la conception qu'avait Adolf Bastian d'une unité fondamentale de l'humanité et de son implication dans un empirisme rigoureux. Déjà, au moment où Franz Boas commença à avoir un certain succès avec ses méthodes aux États-Unis, la jeune génération d'ethnologues allemands, la plupart contemporains de Boas, avait abandonné la vision de Bastian. Ils commençaient à défendre des théories diffusionnistes et à créer des expositions pédagogiques, s'adressant au grand public dans les musées. À l'université de Columbia, cependant, Franz Boas développa et étendit la vision de Bastian au-delà de la culture matérielle et se tourna vers les cultures orales. Il la fit sortir du cadre du musée, étendant la mentalité de collecte de Bastian au-delà des objets des peuples, et mettant l'accent, plus encore, sur « l'enregistrement » de leurs cultures. À travers cet effort, Boas a réalisé en grande partie l'héritage de Bastian.

11 Dans l'atelier de l'après-midi intitulé « Pratiques de patrimonialisation et d'exposition des collections africaines", Musa Oluwaseyi Hambolu (NCMM, Nigéria) a analysé les expositions d'art ancien du Nigéria. Celles-ci peuvent être classées en trois groupes principaux: celles qui concernent seulement les artefacts de la Commission Nationale pour les Musées et Monuments du Nigéria (NCMM), celles qui regroupent à la fois des artefacts dépendants de la NCMM et de collections privées, celles qui présentent les collections de musées étrangers et des collections privées, sans relation avec la NCMM. Bien qu'il n'existe pas toujours de partition très nette entre ces trois groupes, chaque catégorie à ses propres dynamiques et implications. Il est important de mentionner que dans le passé, ces expositions d'art ancien du Nigéria à l'étranger avaient tendance à suivre un cadre national dans leurs compositions. Cependant récemment, les tendances actuelles mettent en exergue des arts traditionnels spécifiques, à l'instar du Bénin, d'IleIfé ou les Nok. On privilégie naturellement ce qui est le plus connu. Deuxièmement, la conception et la préparation de ces projets sont largement menées de l'extérieur. Bien que le degré de participation du Nigéria varie selon les expositions, il y a encore une marge de progrès importante. Troisièmement, il faut aussi mentionner la question de l'impact de ces expositions sur le trafic illégal des antiquités. Si ces expositions sont une vitrine de l'excellence de l'art ancien du Nigéria, nous ne devons pas perdre de vue que ces expositions aiguisent l'appétit des collectionneurs privés, qui n'ont aucun scrupule à bafouer les lois pour acquérir illégalement des antiquités du Nigéria. Quatrièmement, il faut aussi prendre en compte l'avenir des antiquités du Nigéria dans la diaspora. La présentation d'art ancien du Nigéria dans les expositions à l'étranger continuera à susciter de nombreux débats. Ils incombent à tous ceux qui sont concernés de trouver des solutions qui conviennent à tous et de réduire les implications négatives.

Hélène Joubert (Musée du Quai Branly, Paris) a évoqué les arts de la vallée de la Bénoué au Nigéria à propos d'une exposition qu'elle avait organisée au musée du Quai Branly du 12 novembre 2012 au 27 janvier 2013. La région du confluent Niger-Bénoué est depuis plusieurs siècles la terre d'accueil de nombreuses populations, et notamment du nord de la Bénoué, qui emportaient généralement avec elles leurs objets rituels. On y trouve aujourd'hui, entre autres, les Igala, les Ebira, les Idoma, les Afo ou les Tiv. Ces peuples se sont progressivement mélangés pour former de nouvelles communautés, permettant ainsi d'échanger idées et styles artistiques avec leurs voisins. Par exemple, les Tiv se sont déployés à partir du sud, créant un lien culturel avec des peuples ayant partagé une même histoire. Les maternités qui protègent la fertilité humaine et agricole constituent un trait culturel commun aux peuples de cette région. 
Barbara Plankensteiner (Weltmuseum, Vienne) a présenté l'exposition « Bénin. Cinq siècles d'art royal». En 2007 et 2008, le musée d'ethnologie de Vienne a organisé une exceptionnelle exposition internationale sur l'art et l'histoire culturelle du royaume africain occidental en coopération avec trois musées étrangers partenaires. $\mathrm{Y}$ ont participé également la Commission nationale pour les musées et les monuments du Nigéria (NCMM) et l'actuelle Maison Royale de Bénin. Cette contribution a décrit le concept du projet, donné un aperçu des expositions sur les quatre lieux, discuté des difficultés rencontrées, et montré sur le long terme l'impact sur les représentations de la royauté du Bénin dans les musées. Elle a abordé en outre un résultat important de la relation établie au cours de cette coopération qui a conduit à l'établissement d'un dialogue avec le Nigéria, d'une entreprise transcontinentale de coopération en ce qui concerne le patrimoine culturel et le difficile héritage partagé par les musées européens, américains et nigérians.

Nabila Oulebsir (université de Poitiers) et Michaela Oberhofer (Musée Rietberg, Zurich) ont animé la discussion avant que n'ait lieu une table ronde sur les expositions d'art pariétal africain au XXe siècle en Europe et aux États-Unis, à la croisée de l'art et de l'anthropologie avec Thomas Kirchner (Centre allemand d'histoire de l'art), Rémi Labrusse (Paris Ouest-Nanterre), Hélène Ivanoff (EHESS) et Richard Kuba (Institut Frobenius) Le colloque s'est clos avec une conférence de Maria Stavrinaki (Paris 1) à l'Institut Goethe de Paris sur le thème de «La constellation préhistorique autour de Leo Frobenius: Théories et transpositions formelles de l'art pariétal » Les résumés sont accessibles sur ce lien: http://frobenius2014.sciencesconf.org/ resource/page/id/14

\section{AUTEUR}

JEAN-LOUIS GEORGET

(IFHA, Francfort-sur-le-Main) 